\title{
Length and Frequency of bandlimited signals
}

\author{
Jean-Marc Girault, Member, IEEE, Denis Kouamé, Member,IEEE \\ EDIC: SPL.SPTM
}

\begin{abstract}
The main purpose of this letter is to show that measurement of length is an interesting tool to characterize bandlimited signals. We showed the link between the length of a signal and that of the complex instantaneous frequency (CIF). We propose original length and instantaneous frequency estimators which we illustrate by examples.
\end{abstract}

\section{Keywords}

Bandlimited signal, estimator, length, frequency.

\section{INTRODUCTION}

This letter has a dual aim. The first purpose is to familiarise the reader with a technique peripheral to the traditional techniques of signal processing, the second objective is to show that length measurement of bandlimited signals is really an interesting approach.

Although developed at the outset to study signals with the properties of self-similarity or self-affinity such as Tricot chirps (deterministic) or fractional brownian motion (stochastic), we propose applying length measurement to characterize analytic deterministic bandlimited signals.

We therefore initially summarize the length and frequency concepts and then we apply them to the case of analytic deterministic bandlimited signals.

\section{LENGTH AND FREQUENCY CONCEPTS}

\section{A. Length concept}

Measurement of curve lengths, often combined with assessment of fractal dimension, is a major technique to characterize the degree of irregularity of curves or signals [?]. Such length measurement is usually encountered in fields such as the study of traffic on networks, interplanetary magnetic fields, biomedical signals, speech and image compression, to name a few.

The major aspect developed in our study is related to the concept of instantaneous length, also called length elements.

\section{A.1 Length elements and instantaneous velocity}

Having specified beforehand that the signals studied are analytic, it is more convenient initially to express a length element $\overrightarrow{d L_{E}}$ in polar coordinates (see Fig. 1).

The Euclidean length elements are vector $\overrightarrow{d L_{E}}=\overrightarrow{u_{\rho}} d \rho+\rho d \phi \overrightarrow{u_{\phi}}$ and the angular length element vector $\overrightarrow{d \zeta}=\rho d \phi \overrightarrow{u_{\phi}}$ respectively, where $\overrightarrow{u_{\rho}}$ and $\overrightarrow{u_{\phi}}$ are the unitary magnitude and phase vectors respectively, and $d \rho$ and $d \phi$ are the corresponding small elements respectively. The moduli of the two length elements are expressed by:

$$
\left\{\begin{array}{l}
d L_{E}=d \zeta \sqrt{1+\left(\frac{d \rho}{\rho} \frac{1}{d \phi}\right)^{2}} \\
d \zeta=\rho d \phi
\end{array}\right.
$$

We will define thereafter the physical sense of the term $Q=\frac{d \rho}{\rho} \frac{1}{d \phi}$.

The authors work at LUSSI/GIP ULtrasons, Tours, France. E-mail: jmgirault@univ-tours.fr. 
The temporal derivative of this length element is also called instantaneous velocity [?], and can be expressed according to the angular velocity $d \zeta / d t$ as follows:

$$
v(t)=\frac{d L_{E}}{d t}=\frac{d \zeta}{d t} \sqrt{1+Q^{2}}
$$

The length concept can also be considered by calculating the local velocity integral of a signal with a limited support $[0, T]$. Allowing a short-time approach, the latter is expressed by:

$$
L_{E}=\int_{0}^{T} d L_{E}=\int_{0}^{T} v(t) d t=\int_{0}^{T} \rho d \phi \sqrt{1+Q^{2}}
$$

\section{A.2 Example}

Before going into further detail, we analyze a typical example. Let us consider two truncated sinusoids, $x_{1}(t)$ and $x_{2}(t)$. The two signals have the same amplitude and one has twice the frequency of the other: $f_{2}=2 f_{1}$. A geometrical approach, associated with the study of the curves of the two signals, shows that the length of the curve connected to $x_{2}(t)$ seems to be the double that of $x_{1}(t)$ (see Fig. 2).

At this stage, several features must thus be elucidated:

- Does the length of the first curve correspond to double the length of the second curve?

- Are the frequencies and lengths related?

\section{B. Frequency concept}

The frequency concept is often a controversial subject because behind this concept there are three different concepts which are often confused: the concept of frequency which is the inverse of the period ( $f=1 / T)$, the concept of Fourier frequency and instantaneous frequency. We focus here on the temporal definition of the instantaneous frequency of the signal studied $x(t)[?],[?]$.

\section{B.1 Instantaneous frequency}

Instantaneous frequency $\left(f_{i}^{L}(t)\right)$, also called linear instantaneous frequency (LIF), is written as the derivative of the instantaneous phase of the analytic signal $z(t)$ associated with the signal $x(t)$, and applies only to mono-component signals. Linear instantaneous frequency is defined as follows:

$$
f_{i}^{L}(t)=\frac{1}{2 \pi} \frac{d \phi(t)}{d t}
$$

where $\phi(t)$ is the instantaneous phase of the analytic signal. The analytic signal is expressed by:

$$
z(t)=x(t)+j y(t)=\rho(t) e^{j \phi(t)},
$$

where $y(t)=H T(x(t))$ is the Hilbert transform of the signal studied $x(t)$. However, there is a more general definition of instantaneous frequency, i.e. complex instantaneous frequency. As will be seen below, this is the central point of our study.

\section{B.2 Complex instantaneous frequency}

The concept of complex instantaneous frequency (CIF) was introduced by Hahn [?] in order to design oscillators. The CIF $\left(f_{i}^{C}(t)\right)$ is obtained by calculating the temporal derivative of the logarithm of the analytic signal $z(t)$ :

$$
f_{i}^{C}(t)=\frac{1}{2 \pi} \frac{d}{d t}(\ln (z(t)))
$$


After calculation, it is easily demonstrated that the CIF is expressed according to a coefficient $\sigma_{f}(t)$ image of the band-width and according to the LIF:

$$
f_{i}^{C}(t)=\sigma_{f}(t)+j f_{i}^{L}(t)=\left|f_{i}^{C}(t)\right| e^{j \theta(t)},
$$

where

$$
\left\{\begin{array}{l}
\left|f_{i}^{C}(t)\right|=\sqrt{\sigma_{f}^{2}(t)+f_{i}^{L}(t)^{2}}=f_{i}^{L}(t) \sqrt{1+Q^{2}(t)} \\
\theta(t)=\tan ^{-1}(1 / Q(t))
\end{array}\right.
$$

where

$$
\left\{\begin{aligned}
\sigma_{f}(t) & =\frac{1}{2 \pi \rho(t)} \frac{d \rho(t)}{d t} \\
f_{i}^{L}(t) & =\frac{1}{2 \pi \rho(t)} \frac{d \zeta(t)}{d t}
\end{aligned}\right.
$$

and where

$$
Q(t)=\frac{\sigma_{f}(t)}{f_{i}^{L}(t)}=\frac{1}{\rho(t)} \frac{d \rho(t)}{d \phi(t)} .
$$

Note that in the case of narrow band signals $(Q(t) \ll 1$ involving $\theta \rightarrow \pi / 2)$, the LIF can be obtained either by evaluating the modulus of the CIF or directly by taking the imaginary part of the CIF (which is always consistent):

$$
\begin{cases}f_{i}^{L}(t) \approx\left|f_{i}^{C}(t)\right| & \text { if } \mathrm{Q}(\mathrm{t}) \ll 1, \\ f_{i}^{L}(t)=\operatorname{Im}\left(f_{i}^{C}(t)\right) & \forall Q(t),\end{cases}
$$

where $\operatorname{Im}()$ represents the imaginary part.

\section{Relationship between length and frequency}

After having introduced the various concepts, and using (1), (2), (4) it can be seen that the length element $d L_{E}$ is directly connected to the modulus of the CIF:

$$
\frac{d L_{E}(t)}{d t}=2 \pi \rho(t)\left|f_{i}^{C}(t)\right|
$$

In the example above, the length measurement of the two sinusoids (implying $Q(t)=0$ because of its narrow band nature) imposes equality between the LIF and the modulus of the CIF. Furthermore note that under this assumption the signal length is expressed by [?]:

$$
L_{E}(t)=2 \pi T \rho(t) \overline{f_{i}^{L}(t)}
$$

where $\overline{f_{i}^{L}(t)}$ is the instantaneous frequency evaluated over the interval $[0, T]$ corresponding to the duration of the signal studied.

Consequently, eq.(7) confirmes that measuring the length of a narrow band signal is a matter of evaluating the LIF.

\section{ESTIMATORS}

Extending this to evaluation of numerical signals we at the same time propose a length estimator and frequency estimators. We first consider the case of discrete analytic bandlimited signals defined by:

$$
z(n)=x(n)+j y(n)=\rho(n) e^{j \phi(n)}
$$

where $y(n)=H T(x(n))$. 


\section{A. Length estimator}

Several length estimators are described in the literature [?], [?]. Here we will use the generalized Euclidean length element estimator referred to as $d \hat{L}_{E}(n, k)$ and expressed by:

$$
d \hat{L}_{E}(n, k)=\frac{|z(n+k)-z(n)|}{k}=\frac{1}{k}\left|\sum_{p=1}^{k} z(n+p)-z(n+p-1)\right|
$$

where $k$, referred to as a gauge, plays the role of zoom and $n$ is the current time.

From eq.(8) several comments can be made:

- Eq.(8) shows a local average operation of the increment function $(z(n+p)-z(n+p-1))$. Note that $\hat{L}_{E}(n, k)=\sum_{i=1}^{n} d \hat{L}_{E}(i, k)$.

- Eq.(8) is an extended version of the classical Euclidean length element estimator: $d \hat{L}_{E}(n)=\mid z(n+1)-z(n)$

- In the case of analytic signals, estimators proposed by Higuchi [?], Burlaga and Klein [?] are a global version of eq.(8)except for a coefficient $1 / k$.

Knowing that the increment function $(z(n+k)-z(n))$ is a first order interpolation operation (straight line between two consecutive samples), length measurement is equivalent to a low-pass filter operation. Note that measuring $d \hat{L}_{E}(n, k)$ comes down to evaluating $d \hat{L}_{E}(n)$ at a rate of $1 / k$ times the original sample rate, this is a decimation operation. Finally, measured length is not that of the studied signal, but that of a decimated and first-order interpolated signal (low-pass filter). Although we chose to limit ourselves to a first-order interpolation polynomial, this interpolation can obviously also be extended to higher orders or even to other families of interpolation polynomials.

\section{B. Frequency estimators}

In the case of discrete signals eq.(6) can be rewritten taking into account the estimate of the complex instantaneous frequency:

$$
\left|\hat{f}_{i}^{C}(n, k)\right|=\frac{1}{2 \pi \rho(n)} \frac{d \hat{L}_{E}(n, k)}{T_{s}}
$$

where $T_{s}=1 / f_{s}$ is the sampling period. The estimator $\left|\hat{f}_{i}^{C}(n, k)\right|$ can be written in the same form as in eq.(4):

$$
\left|\hat{f}_{i}^{C}(n, k)\right|=\sqrt{\left(\hat{\sigma}_{f}(n, k)\right)^{2}+\left(\hat{f}_{i}^{L}(n, k)\right)^{2}},
$$

where (see Appendix)

$$
\left\{\begin{array}{l}
\hat{\sigma}_{f}(n, k)=\frac{1}{2 \pi k T_{s}}\left(1-\frac{\rho(n+k)}{\rho(n)}\right), \\
\hat{f}_{i}^{L}(n, k)=\frac{1}{\pi k T_{s}} \sqrt{\frac{\rho(n+k)}{\rho(n)}} \mid \sin \left(\pi k T_{s} \overline{\left.\hat{f}_{i}^{L}(n, k)\right)} \mid\right.
\end{array}\right.
$$

and where $\overline{\hat{f}_{i}^{L}(n, k)}$ expresses an average frequential quantity evaluated from the last $k$ values (see appendix).

It should be noted that:

1) by converging to the continuous case, i.e. by considering the high sampling frequency $\left(f_{s} \rightarrow \infty\right)$ compared with the highest frequency of the signal, eq.(10) becomes:

$$
\lim _{f_{s} \rightarrow \infty}\left|\hat{f}_{i}^{C}(n, k)\right|=\sqrt{\hat{\sigma}_{f}^{2}(n, k)+\left(\overline{\hat{f}_{i}^{L}(n, k)} \sqrt{\frac{\rho(n+k)}{\rho(n)}}\right)^{2}},
$$


2) from 1) and using the minimum integer value of the gauge $k=1$, eq.(10) tends to eq.(4):

$$
\lim _{f_{s} \rightarrow \infty}\left|\hat{f}_{i}^{C}(n, 1)\right|=\sqrt{\hat{\sigma}_{f}^{2}(n, 1)+\hat{f}_{i}^{L}(n, 1)^{2}}
$$

where $\hat{f}_{i}^{L}(n, 1)=f_{i}^{L}(n+1) \sqrt{\frac{\rho(n+1)}{\rho(n)}}$.

Note that $\hat{\sigma}_{f}(n, 1)$ is exactly the discrete form of $\sigma_{f}(t)$ whereas $\hat{f}_{i}{ }^{L}(n, 1)$ is only for a narrow band hypothesis: $\hat{f}_{i}^{L}(n, 1) \approx f_{i}^{L}(n+1)$.

3) By considering the narrow band assumption only, i.e. $\rho(n+k) / \rho(n) \approx 1 \Rightarrow \hat{\sigma}_{f}(n, k) \approx 0$, eq.(10) becomes:

$$
\left|\hat{f}_{i}^{C}(n, k)\right| \approx \overline{\hat{f}_{i}^{L}(n, k)} \mid \operatorname{sinc}\left(\pi k T_{s} \overline{\left.\hat{f}_{i}^{L}(n, k)\right)} \mid\right. \text {. }
$$

Owing to the periodic nature of eq. $(11),\left|\hat{f}_{i}^{C}(n, k)\right|=0$ for any $\hat{k}_{\alpha}(n)=\alpha f_{s} / \overline{\hat{f}_{i}^{L}(n)}$ with $\alpha=1,2,3, \ldots$. Therefore it is possible to evaluate the mean instantaneous frequency by detecting zero-crossings. In particular, estimation of the first minimum of the eq.(11), i.e. $\hat{k}_{1}(n)=\min \left(\left\{k / \hat{f}_{i}^{C}(n, k)=0\right\}\right)$, also leads to:

$$
\overline{\hat{f}_{i}^{L}(n)}=\frac{f_{s}}{\hat{k}_{1}(n)}
$$

This is the first estimator of the mean instantaneous frequency. As it has been suggested above, this can be extended to the detection of several zero-crossings and even provide an additional average.

4) by considering 2 ) and 3), eq.(10) becomes:

$$
\lim _{f_{s} \rightarrow \infty}\left|\hat{f}_{i}^{C}(n, 1)\right|=f_{i}^{L}(n+1) .
$$

The latter estimate which constitutes our second estimator is the discret form of eq.(5).

\section{Examples}

To illustrate the behavior of the proposed estimators (eq.(12), eq.(13)), we consider two examples: - A complex sinusoid embedded in a white complex noise $\eta$ for different SNR, defined by: $z(t)=$ $A \exp \left(2 \pi j f_{i}^{L} t\right)+\eta(t)$.

- A bandlimited FM signal (gaussian envelope and sinusoidal frequential law) defined by: $z(t)=$ $\exp \left(-\left(t-t_{0}\right)^{2} /\left(2 \sigma_{0}^{2}\right)\right) \exp \left(2 \pi j t f_{c}+j \beta \cos \left(2 \pi t f_{0}\right)\right)$, where $t_{0}=0.5 \mathrm{~s}, \beta=40 \pi, \sigma_{0}=0.0194$, and where $f_{0}=4 \mathrm{~Hz}, f_{c}=1500 \mathrm{~Hz}$ and $f_{s}=10000 \mathrm{~Hz}$.

By way of illustration, Fig. 3, presents the evolution of $\left|\hat{f}_{i}^{C}(n, k)\right|(9)$ or $d \hat{L}_{E}(n, k)$ (8) in the case of sinusoids embedded in a white noise. It can be seen in Fig. 3 that for weak SNR the second estimator $\hat{f}_{i}^{C}(n, 1)$ is strongly biased whereas the first is not. Moreover it is shown that the first estimator is less sensitive to noise than the second because it represents an average estimate of instantaneous frequency. In addition, Fig. 3 shows that for weak SNR $(<-5 d B)$, neither estimator provides a more precise value of the frequency. Finally, Fig. 4 shows the sinusoid law of the FM signal (gaussian envelope) for both estimators.

In the case of filtered random signals [?], for example $1 / f^{\alpha}$ noise, it is possible to establish the link between deterministic and random narrow band signals and to provide additional information on fractal features. 


\section{CONCLUSION}

In conclusion, we have shown that a simple geometrical approach makes it possible to illustrate the dependence existing between the length of a signal and its complex instantaneous frequency. Furthermore, we have demonstrated here that it is possible to estimate the average instantaneous frequency by length measurement.

\section{APPENDIX}

By using eqs.(8),(9), eq.(10) becomes:

$$
\left|\hat{f}_{i}^{C}(n, k)\right|=\frac{\left|\rho(n)-\rho(n+k) e^{j \Delta \phi}\right|}{2 \pi k T_{s} \rho(n)},
$$

where $\Delta \phi=\phi(n+k)-\phi(n)$.

$$
\left|\hat{f}_{i}^{C}(n, k)\right|=\sqrt{\left(\frac{\Delta_{\rho}}{2 \pi T_{s} \rho(n)}\right)^{2}+\left(\sqrt{\frac{\rho(n+k)}{\rho(n)}} \frac{\sin (\Delta \phi / 2)}{\pi k T_{s}}\right)^{2}},
$$

where $\Delta_{\rho}=\rho(n+k)-\rho(n)$, with

$$
\Delta \phi / 2=\pi T_{s} \overline{\hat{f}_{i}^{L}(n, k)}=\pi T_{s}\left(\sum_{l=0}^{n+k} f_{i}^{L}(l)-\sum_{l=0}^{n} f_{i}^{L}(l)\right),
$$

and where $\overline{\hat{f}_{i}^{L}(n, k)}=\frac{1}{k} \sum_{l=1}^{k} f_{i}^{L}(n+l)$. 


\section{List OF FiguRES}

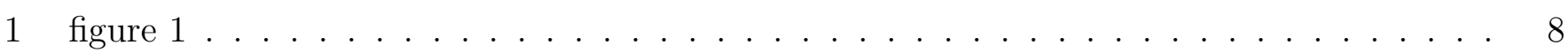

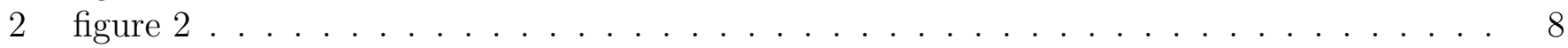

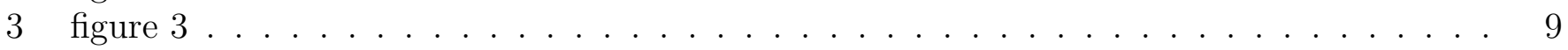

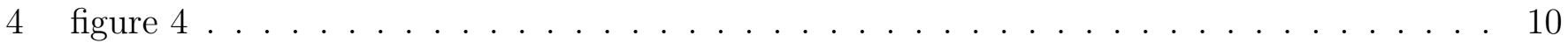

\section{List OF Figures}


a)

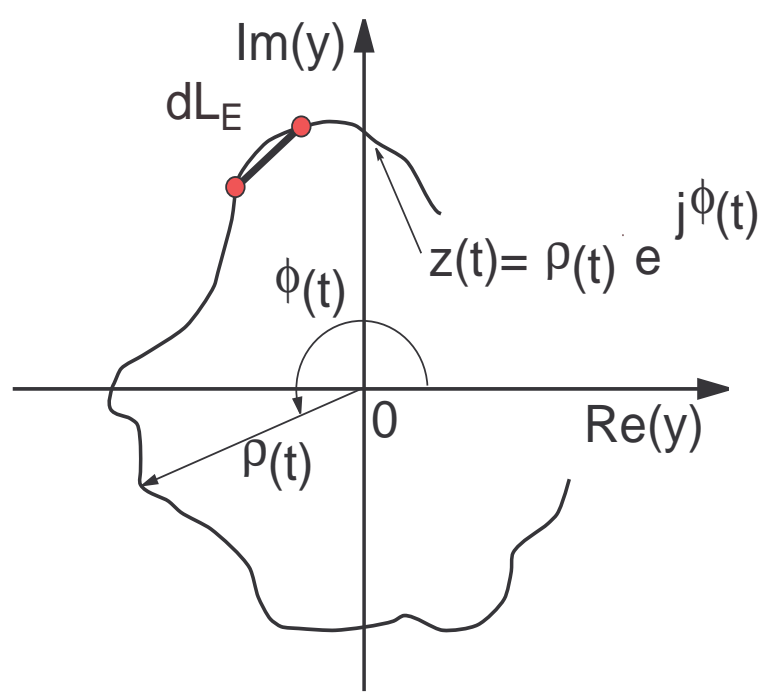

b)

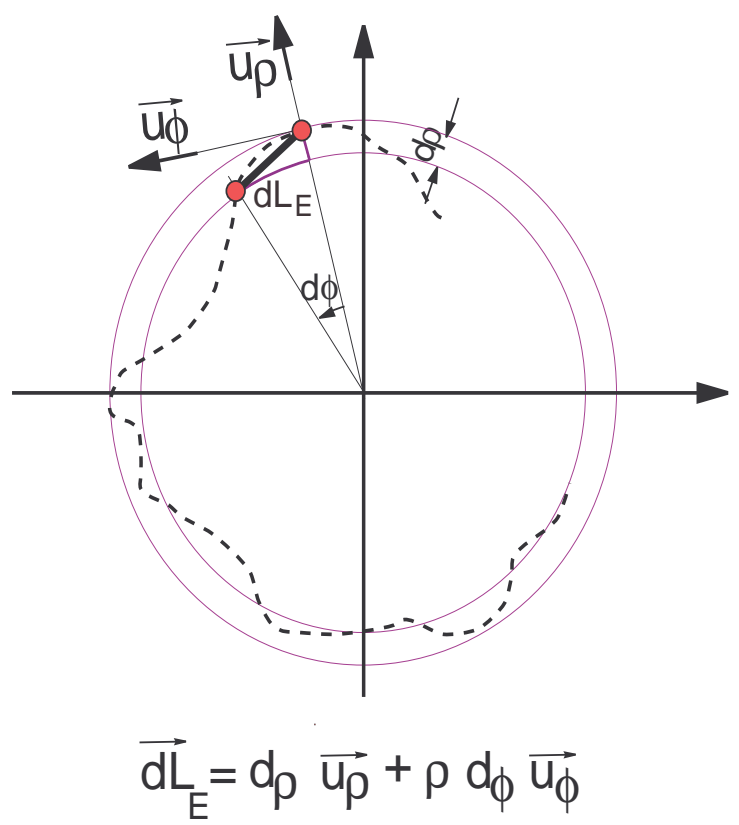

Fig. 1.

Polar representations. a) Polar representation of a modulated signal. b) Polar representation of a length element.

SIGNAL

LENGTH

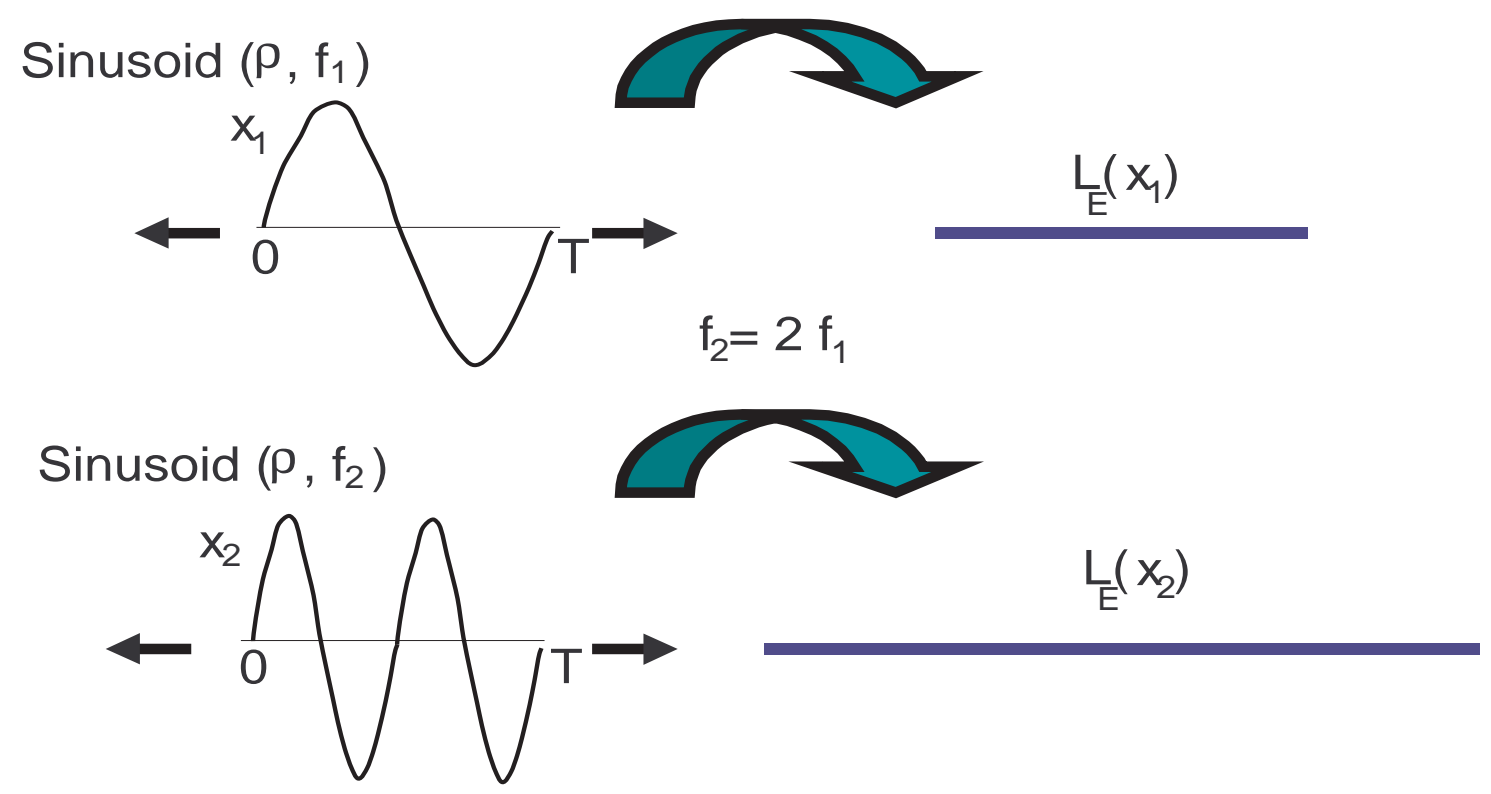

Fig. 2.

Lengths of two sinusoids of same amplitude and different frequencies. 


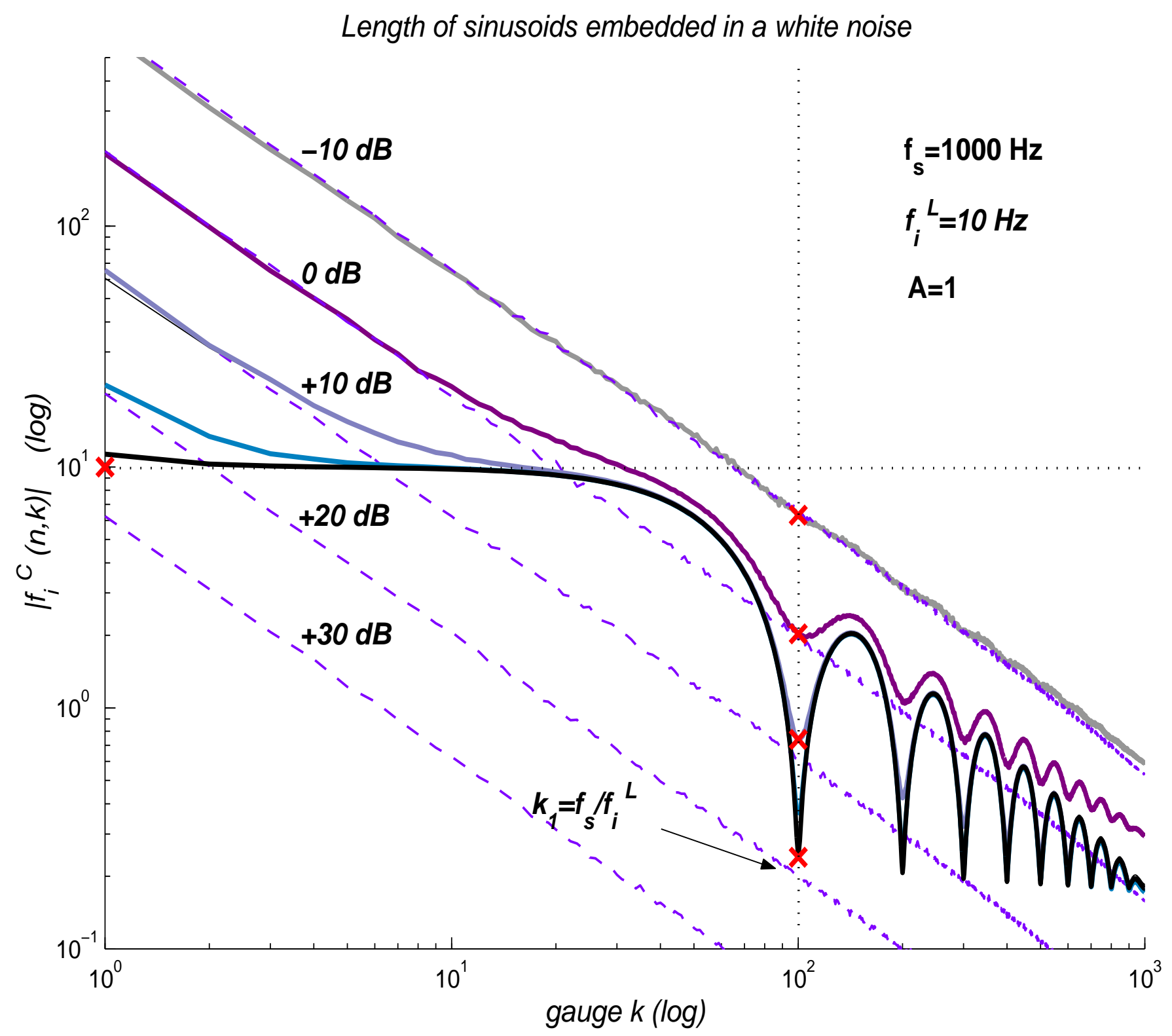

Fig. 3.

Instantaneous frequency estimate for sinusoids embedded in white noise for various $S N R$ ( $-10 d B$, up to $30 \mathrm{~dB}$ ). The solid line curves are disturbed signals whereas the dotted lines represent only the white noise. 


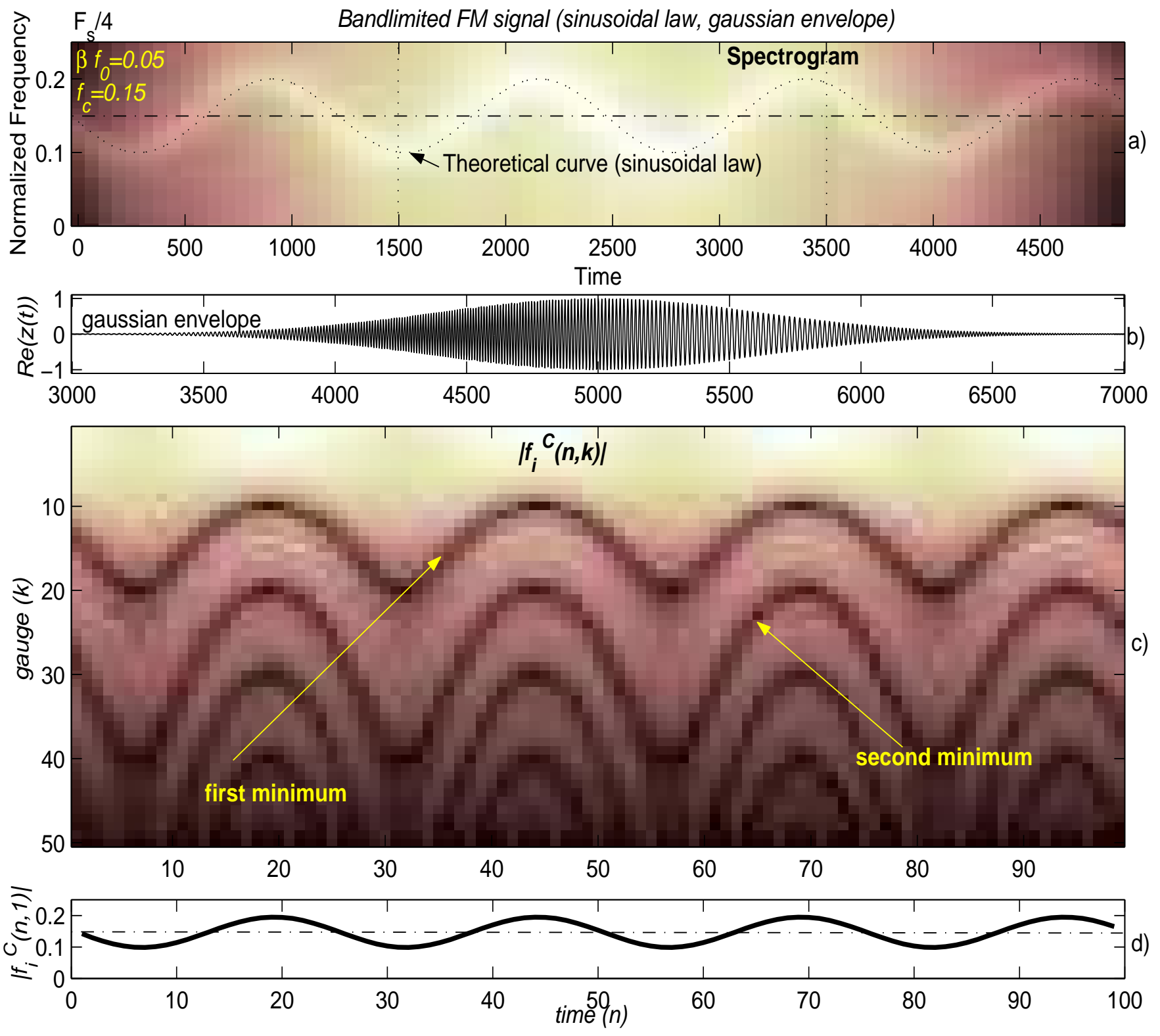

Fig. 4.

Frequency estimation of a bandlimited FM signal. a) Spectrogram of the analyzed signal, b) zoom of the real part of the analytic signal $z(t)$ (noted $\operatorname{Re}(z(t))$ ) delimited in the spectrogram, c) first estimator: Bidimensional plan of $\left|\hat{f}_{i}^{C}(n, k)\right|$, d) second estimator $\left|\hat{f}_{i}^{C}(n, 1)\right|$. 